PROCEEDINGS OF THE

AMERICAN MATHEMATICAL SOCIETY

Volume 140, Number 5, May 2012, Pages 1709-1717

S 0002-9939(2011)11019-0

Article electronically published on September 6, 2011

\title{
MINIMAL VOLUME OF SLABS IN THE COMPLEX CUBE
}

\author{
HERMANN KÖNIG AND ALEXANDER KOLDOBSKY \\ (Communicated by Thomas Schlumprecht)
}

\begin{abstract}
We study the volume of sections and slabs in the $n$-dimensional cube for complex scalars. In particular, we investigate the directions of minimal volume for a small width of the slab.
\end{abstract}

\section{INTRODUCTION AND RESULTS}

Let $\|\cdot\|_{\infty}$ and $|\cdot|$ denote the supremum and the euclidean norm on $\mathbb{K}^{n}$ where $\mathbb{K} \in\{\mathbb{R}, \mathbb{C}\}$. For volume considerations, $\mathbb{C}^{n}$ is identified with $\mathbb{R}^{2 n}$ and the $(2 n)$ dimensional volume is used. Let

$$
B_{\infty}:=\left\{x \in \mathbb{K}^{n} \mid\|x\|_{\infty} \leq \alpha\right\}
$$

be the $n$-dimensional cube of volume 1, i.e. $\alpha=1 / 2$ if $\mathbb{K}=\mathbb{R}$ and $\alpha=1 / \sqrt{\pi}$ if $\mathbb{K}=\mathbb{C}$. Given some direction $a \in \mathbb{K}^{n}$ of length $|a|=1$ and $t \in \mathbb{K}$, we introduce the section of the cube

$$
S(a, t):=\left\{x \in \mathbb{K}^{n} \mid\|x\|_{\infty} \leq \alpha,\langle x, a\rangle=\alpha t\right\}
$$

and for $t>0$ the slab in the cube

$$
S \ell(a, t):=\left\{x \in \mathbb{K}^{n}\left|\|x\|_{\infty} \leq \alpha,\right|\langle x, a\rangle \mid \leq \alpha t\right\} .
$$

Let

$$
\begin{aligned}
A(a, t):=\operatorname{vol}_{n-1}(S(a, t)) & , \quad t \in \mathbb{K}, \\
V(a, t):=\operatorname{vol}_{n}(S \ell(a, t)) & , \quad t>0,
\end{aligned}
$$

denote the volumes of the section and the slab of $B_{\infty}$. In the complex case, one has to use $\operatorname{vol}_{2 n-2}$ and $\operatorname{vol}_{2 n}$ for $A$ and $V$. For $a=\left(a_{k}\right)_{k=1}^{n}$, let $a^{*}$ denote the decreasing rearrangement of the sequence $\left(\left|a_{k}\right|\right)_{k=1}^{n}$, i.e. $\left|a_{1}\right| \geq \cdots \geq\left|a_{n}\right| \geq 0$. Since the volume is invariant under coordinate permutations and sign changes, which in the complex case means invariant under permutations and rotations of coordinate disks, we have

$$
A(a, t)=A\left(a^{*},|t|\right), V(a, t)=V\left(a^{*}, t\right) .
$$

We will therefore assume in the following that $a=\left(a_{k}\right)_{k=1}^{n}, a_{1} \geq \cdots \geq a_{n} \geq 0$ and $t \geq 0$

Received by the editors August 26, 2010 and, in revised form, January 21, 2011.

2010 Mathematics Subject Classification. Primary 46B20, 52A20, 52A40; Secondary 46B04, $52 \mathrm{~A} 21$.

The first author was partially supported by the American Institute of Mathematics.

The second author was partially supported by NSF grant DMS-1001234 and the American Institute of Mathematics.

(C)2011 American Mathematical Society
Reverts to public domain 28 years from publication 1709 
Let $f_{1}=(1,0, \ldots, 0), f_{2}:=(1,1,0, \ldots, 0) / \sqrt{2}$. For central sections $1=A\left(f_{1}, 0\right)$ $\leq A(a, 0) \leq A\left(f_{2}, 0\right)=2^{m / 2}$, and where $m=1$ for $\mathbb{K}=\mathbb{R}, m=2$ for $\mathbb{K}=\mathbb{C}$. The lower bound is due to Hadwiger [Ha], Vaaler [V] and Hensley [He] the upper bound for $\mathbb{K}=\mathbb{R}$ is a well-known result of $\mathrm{K}$. Ball $[\mathrm{B}$. The complex case is due to Oleskiewicz-Pełczyński OP.

For small $t>0$ it would seem natural that the volume of slabs mimics this behaviour in the sense that for $\mathbb{K}=\mathbb{R}$

$$
t=V\left(f_{1}, t\right) \leq V(a, t) \leq V\left(f_{2}, t\right)=\sqrt{2} t-t^{2} / 2 .
$$

In fact, it was proved by Barthe-Koldobsky $\mathrm{BK}$ that the lower bound is true provided that $t \leq 3 / 4$ holds. For $t>2(\sqrt{2}-1)$, however, $V\left(f_{1}, t\right) \leq V(a, t)$ is false for $a=f_{2}$, and for $t$ larger but close to this number $2(\sqrt{2}-1), f_{2}$ probably is the direction of minimal slab volume (this is true at least for $n=2$ and 3). The lower bound

$$
t^{2}=V\left(f_{1}, t\right) \leq V(a, t)
$$

remains true in the complex case for small $t$. This generalization of the result of BK to the complex case is the main result of this paper. Formula (2.3) states that

$$
V\left(f_{2}, t\right)=2 t^{2}-\left(\left(1+t^{2}\right) t \sqrt{2-t^{2}}+\left(2 t^{2}-1\right) \arcsin \left(t \sqrt{2-t^{2}}\right)\right) / \pi,
$$

and for $t \geq t_{1} \approx 0.867115, V\left(f_{2}, t\right)<V\left(f_{1}, t\right)$, and for $t \geq t_{1}$ but near $t_{1}, t_{2}$ is probably the direction of minimal volume (true for $n=2$ ). Our main result states that $f_{1}$ is the direction of minimal volume for slabs of width $t \leq 4 / 5$, leaving a gap for width $4 / 5<t<t_{1}$ similar to the real case where the gap is $3 / 4<t<2(\sqrt{2}-1)$.

Theorem 1. Let $\mathbb{K}=\mathbb{C}, a \in \mathbb{C}^{n}$ with $|a|=1$ and $0 \leq t \leq 4 / 5$. Then

$$
t^{2}=V\left(f_{1}, t\right) \leq V(a, t) .
$$

Note that

$$
V(a, t)=2 \int_{0}^{t} A(a, s) s d s, 1=\operatorname{vol}_{2 n}\left(B_{\infty}\right)=2 \int_{0}^{\infty} A(a, s) s d s .
$$

As in $[\mathrm{BK}$ the main technical tool is an integral inequality of independent interest, to be applied to $f=2 A(a, \cdot)$ :

Proposition 2. Let $f: \mathbb{R}_{+} \rightarrow \mathbb{R}_{+}$be in $L_{1}\left(\mathbb{R}_{+}\right.$, sds), non-increasing and logconcave. Let $t>0$. Assume that

$$
\int_{0}^{t} f(s) s d s \leq\left(\frac{4}{5}\right)^{2} \int_{0}^{\infty} f(s) s d s .
$$

Then

$$
2\left(\int_{0}^{\infty} f(s) s^{3} d s\right)\left(\int_{0}^{t} f(s) s d s\right) \geq t^{2}\left(\int_{0}^{\infty} f(s) s d s\right)^{2} .
$$

Concerning the direction of the maximal volume of slabs, in the case of complex scalars, different from the case of the maximal volume of sections [OP], $V(a, t) \leq$ $V\left(f_{2}, t\right)$ is false in general for any width $t>0$ : for large $n \in \mathbb{N}$, we show $V\left(f_{n}, t\right)>$ $V\left(f_{2}, t\right)$, where $f_{n}:=(1, \ldots, 1) / \sqrt{n} \in S^{n-1}$ : 
Proposition 3. Let $0<t<4 / 9$ and $n \geq 20$. Then for $\mathbb{K}=\mathbb{C}$ and any $n \geq 1 / t$,

$$
V\left(f_{n}, t\right)>V\left(f_{2}, t\right) .
$$

Contrary to this, in the case of real scalars $\mathbb{K}=\mathbb{R}, V\left(f_{n}, t\right)<V\left(f_{2}, t\right)$ holds provided that $t<t_{0}$, where $t_{0}$ is approximately $1 / 14$.

\section{Volume FORMulas}

We mention and recall some volume formulas for sections and slabs of the cube which yield some statements and formulas before the formulation of Theorem 1.

Proposition 4. Let $a \in \mathbb{R}_{+}^{n},|a|=1, t \geq 0$. Then

$$
A(a, t)=\left\{\begin{array}{ll}
\frac{2}{\pi} \int_{0}^{\infty} \prod_{k=1}^{n} \frac{\sin \left(a_{k} s\right)}{a_{k} s} \cos (t s) d s & \mathbb{K}=\mathbb{R} \\
\frac{1}{2} \int_{0}^{\infty} \prod_{k=1}^{n} j_{1}\left(a_{k} s\right) J_{0}(t s) s d s & \mathbb{K}=\mathbb{C}
\end{array}\right\}
$$

and

$$
V(a, t)=\left\{\begin{array}{lll}
t \frac{2}{\pi} \int_{0}^{\infty} \prod_{k=1}^{n} \frac{\sin \left(a_{k} s\right)}{a_{k} s} \frac{\sin (t s)}{t s} d s & \mathbb{K}=\mathbb{R} \\
t^{2} \frac{1}{2} \int_{0}^{\infty} \prod_{k=1}^{\infty} j_{1}\left(a_{k} s\right) j_{1}(t s) s d s & \mathbb{K}=\mathbb{C}
\end{array}\right\},
$$

where $J_{0}, J_{1}$ are the first Bessel functions and $j_{1}(x)=2 J_{1}(x) / x$.

Let us note that (2.1), $\mathbb{K}=\mathbb{R}$ is a well-known result of Polya $[\mathrm{P}$. For $\mathbb{K}=\mathbb{C}$ and $t=0$, it is found in $\mathrm{OP}$. Formulas (2.2) follow from (2.1) by integrating over $t$, using $J_{0}(s) s=\left(J_{1}(s) s\right)^{\prime}$ in the complex case. The standard way to prove (2.1) is to write (with $\alpha$ as in the introduction)

$$
A(a, t)=\int_{\langle z, a\rangle=\alpha t} \chi\left(\|z\|_{\infty}\right) d z,
$$

where $\chi$ is the indicator function of $[0, \alpha]$, and to take the Fourier transform in the $t$-variable, $\hat{A}(a, \cdot)$, which by coordinate independence is a constant times a product of scaled (by $a_{k}$ ) Fourier transforms of the ball $B=\{z \in \mathbb{K}|| z \mid \leq \alpha\}$. For $\mathbb{K}=\mathbb{C}$ this is where $J_{1}$ appears. Taking the inverse transform then gives (2.1).

In particular, for $a=f_{1}$ and $a=f_{2},(2.2)$ yields

$$
\begin{aligned}
& V\left(f_{1}, t\right)=t \quad(\mathbb{K}=\mathbb{R}), V\left(f_{1}, t\right)=t^{2} \quad(\mathbb{K}=\mathbb{C}), \\
& V\left(f_{2}, t\right)=\sqrt{2} t-t^{2} / 2 \quad(\mathbb{K}=\mathbb{R}), \\
& V\left(f_{2}, t\right)=2 t^{2}-\left(\left(1+t^{2}\right) t \sqrt{2-t^{2}}+\left(2 t^{2}-1\right) \arcsin \left(t \sqrt{2-t^{2}}\right)\right) / \pi \quad(\mathbb{K}=\mathbb{C})
\end{aligned}
$$

so that $V\left(f_{1}, t_{1}\right)=V\left(f_{2}, t_{1}\right)$ for $t_{1}=2(\sqrt{2}-1)$ in the real case and $t_{1} \simeq 0.867115$ in the complex case, as mentioned in the introduction. For $0<t<t_{1}, V\left(f_{1}, t\right)<$ $V\left(f_{2}, t\right)$.

Using these formulas, we now give the 
Proof of Proposition 3. (a) For $f_{n}=(1, \ldots, 1) / \sqrt{n}$, (2.2) implies for complex scalars and $t>0$ :

$$
V\left(f_{n}, t\right)=\frac{t^{2}}{2} \int_{0}^{\infty}\left(j_{1}(s / \sqrt{n})\right)^{n} j_{1}(t s) s d s .
$$

Since $\lim _{u \rightarrow 0} j_{1}(u) e^{u^{2} / 8}=1, \lim _{n \rightarrow \infty} j_{1}(s / \sqrt{n})^{n}=e^{-s^{2} / 8}$ for any $s \in \mathbb{R}$, and we find using the dominated convergence theorem that

$$
\begin{aligned}
\lim _{n \rightarrow \infty} V\left(f_{n}, t\right) & =\frac{t^{2}}{2} \int_{0}^{\infty} e^{-s^{2} / 8} j_{1}(t s) s d s \\
& =t \int_{0}^{\infty} e^{-s^{2} / 8} J_{1}(t s) d s=1-e^{-2 t^{2}} .
\end{aligned}
$$

(b) For the properties of Bessel functions, cf. Watson [W].

We will use the following estimates for the modified Bessel functions $j_{1}(t)=$ $2 J_{1}(t) / t$ :

$$
\begin{aligned}
& \left|j_{1}(t)\right| \leq \min \left(1,1.66 / t^{3 / 2}\right), \quad 0<t, \\
& \left|j_{1}(t)\right| \geq \exp \left(-t^{2} / 8-t^{4} / 316\right), \quad 0 \leq t \leq 2 .
\end{aligned}
$$

As for $(2.5), \sqrt{t}\left|J_{1}(t)\right|$ has a decreasing sequence of maxima, tending to $\sqrt{2 / \pi}$ by the asymptotic formula for $J_{1}(t)$, with the first maximum near $t_{0} \approx 2.17$ bounded by 0.83 . To prove (2.6), consider $f(t):=j_{1}(t) \exp \left(t^{2} / 8+t^{4} / 316\right)$. Then

$$
g(t):=f^{\prime}(t) \exp \left(-t^{2} / 8-t^{4} / 316\right)=J_{1}(t)\left(1 / 2+2 / 79 t^{2}\right)-2 J_{2}(t) / t .
$$

In $[0,2], g$ is positive for $t<t_{1} \approx 1.67$ and negative for $t>t_{1}$, as can be checked by using the alternating power series expansion of $J_{1}$ and $J_{2}$ to approximate $g$ by polynomials. Thus $f$ is first increasing and then decreasing. Since $f(0)=1$ and $f(2)>0,(2.6)$ holds for $t \in[0,2]$.

(c) By (2.2) and (2.4), we have for any $t>0$

$$
V\left(f_{n}, t\right)=\left(1-e^{-2 t^{2}}\right)-t^{2} / 2 \int_{0}^{\infty}\left(e^{-s^{2} / 8}-j_{1}(s / \sqrt{n})^{n}\right) j_{1}(t s) s d s .
$$

Using (2.6) and $\left|j_{1}(u)\right| \leq 1$, we find

$$
\begin{aligned}
I_{1} & :=t^{2} / 2 \int_{0}^{2 \sqrt{n}}\left|e^{-s^{2} / 8}-j_{1}(s / \sqrt{n})^{n}\right| j_{1}(t s) s d s \\
& \leq t^{2} / 2 \int_{0}^{2 \sqrt{n}} e^{-s^{2} / 8}\left(1-e^{-s^{4} /(316 n)}\right) s d s \\
& \leq t^{2} / 2 \int_{0}^{\infty} e^{-s^{2} / 8} s^{5} /(316 n) d s=64 / 79 t^{2} / n .
\end{aligned}
$$

For $s>2 \sqrt{n}$, we estimate both terms in the integrand in (2.7) separately:

$$
I_{2}:=t^{2} / 2 \int_{2 \sqrt{n}}^{\infty} e^{-s^{2} / 8}\left|j_{1}(t s)\right| s d s \leq t^{2} / 2 \int_{2 \sqrt{n}}^{\infty} s e^{-s^{2} / 8} d s=2 / e^{n / 2} t^{2}
$$


and, using (2.5),

$$
\begin{aligned}
I_{3} & :=t^{2} / 2 \int_{2 \sqrt{n}}^{\infty}\left|j_{1}(s / \sqrt{n})\right|^{n}\left|j_{1}(t s)\right| s d s=t^{2} n / 2 \int_{2}^{\infty}\left|j_{1}(u)\right|^{n}\left|j_{1}(\sqrt{n} t u)\right| u d u \\
& \leq t^{2} n / 2 \int_{2}^{\infty}\left(1.66 / u^{3 / 2}\right)^{n} u d u=4 n /(3 n-4)(0.83 / \sqrt{2})^{n} t^{2} \leq 2 / e^{n / 2} t^{2}, \quad n \geq 4 .
\end{aligned}
$$

Therefore $I:=I_{1}+I_{2}+I_{3} \leq\left(64 / 79+4 n / e^{n / 2}\right) t^{2} / n \leq 0.815 t^{2} / n$, provided that $n \geq 20$. Using formula $(2.3)$ for $V\left(f_{2}, t\right)$, elementary estimates give that $V\left(f_{2}, t\right) \leq$ $2 t^{2}-t^{3}$ for any $t \in[0,1]$. Therefore $V\left(f_{2}, t\right)<V\left(f_{n}, t\right)$ will follow from $0.815+$ $2 n t^{2}<n t$. It is elementary to check that this holds for $1 / n<t<4 / 9$ and $n \geq 20$.

\section{Slabs of minimal VOlume}

We now prove Theorem 1 by first using Proposition 2 and then giving the (independent) proof of Proposition 2.

Proof of Theorem 1 from Proposition 2. Let $a \in S^{n-1}(\mathbb{C})$ and $0 \leq t \leq 4 / 5$. For the volume of the slab and the unit cube we have in the complex case by polar integration

$$
V(a, t)=2 \int_{0}^{t} A(a, s) s d s, 1=2 \int_{0}^{\infty} A(a, s) s d s .
$$

Assume that the direction $a$ satisfies $V(a, t) \leq V\left(f_{1}, t\right)=t^{2}$. We claim that in this case $V(a, t)=V\left(f_{1}, t\right)$; i.e. $f_{1}$ is a minimal volume direction for the complex slab. The assumption in Proposition 2 is satisfied for $f=2 A(a, \cdot)$ since

$$
V(a, t)=\int_{0}^{t} f(s) s d s \leq t^{2} \leq\left(\frac{4}{5}\right)^{2}=\left(\frac{4}{5}\right)^{2} \int_{0}^{\infty} f(s) s d s .
$$

Further, for the circle $B=\{z \in \mathbb{C}|| z \mid \leq \alpha=1 / \sqrt{\pi}\}$ of area 1,

$$
\begin{aligned}
\int_{0}^{\infty} f(s) s^{3} d s & =2 \int_{0}^{\infty} A(a, s) s^{3} d s \\
& =2 \pi^{2} \int_{0}^{\infty} A(a, u / \alpha) u^{3} d u \\
& =\pi \int_{B} A(a,|v| / \alpha)|v|^{2} d v \\
& =\pi \int_{B}\left(\int_{\langle z, a\rangle=|v|} d z\right)|v|^{2} d v \\
& =\pi \int_{B_{\infty}}|\langle w, a\rangle|^{2} d w=\frac{1}{2},
\end{aligned}
$$


where the last equality follows from

$$
\int_{B^{2}} w_{i} \bar{w}_{j} d w_{i} d w_{j}=0, \int_{B}\left|w_{i}\right|^{2} d w_{i}=\frac{1}{2 \pi} .
$$

We thus conclude by Proposition 2

$$
2\left(\int_{0}^{\infty} f(s) s^{3} d s\right)\left(\int_{0}^{t} f(s) s d s\right) \geq t^{2}\left(\int_{0}^{\infty} f(s) s d s\right)^{2} ;
$$

i.e. $2 \cdot \frac{1}{2} \cdot V(a, t) \geq t^{2} \cdot 1^{2}, V(a, t) \geq t^{2}=V\left(f_{1}, t\right)$.

Proof of Proposition 2. Let $t>0$ and $0<v<V$. Consider

$$
\begin{aligned}
F_{t, v, V}:=\left\{f: \mathbb{R}_{+} \rightarrow \mathbb{R}_{+} \mid f\right. & \\
& \text { non-increasing, log-concave, } \left.\int_{0}^{\infty} f(s) s d s=V, \int_{0}^{t} f(s) s d s=v\right\} .
\end{aligned}
$$

We want to prove that under the assumption (1.2) on $f$,

$$
\inf \left\{\int_{0}^{\infty} f(s) s^{3} d s \mid f \in F_{t, v, V}\right\} \geq \frac{t^{2} V^{2}}{2 v} .
$$

Using the monotonicity and $\log$-concavity of $f$, one proves similarly as in BK] that

$$
\begin{aligned}
& \inf \left\{\int_{0}^{\infty} f(s) s^{3} d s \mid f \in F_{t, v, V}\right\} \\
& =\inf \left\{\int_{0}^{d} e^{-p s} s^{3} d s \mid p \geq 0, d \geq t \geq 0, \int_{0}^{d} e^{-p s} s d s=V, \int_{0}^{t} e^{-p s} s d s=v\right\} .
\end{aligned}
$$

Thus we only have to consider functions $f(s)=\chi_{[0, d]}(s) e^{-p s}$. The constraints in (3.2) mean that

$$
V=\int_{0}^{d} e^{-p s} s d s=\frac{1}{p^{2}} \int_{0}^{x} e^{-u} u d u, v=\int_{0}^{t} e^{-p s} s d s=\frac{1}{p^{2}} \int_{0}^{y} e^{-u} u d u,
$$

with $x:=p d \geq y:=p t$. Moreover,

$$
\int_{0}^{d} e^{-p s} s^{3} d s=\frac{1}{p^{4}} \int_{0}^{x} e^{-u} u^{3} d u
$$

Assumption (1.2) for these functions is thus equivalent to

$$
\int_{0}^{y} e^{-u} u d u \leq\left(\frac{4}{5}\right)^{2} \int_{0}^{x} e^{-u} u d u, 0 \leq y \leq x .
$$


Therefore, using (3.2), inequality (1.3) of Proposition 2 will follow provided we can show that for any $0 \leq y \leq x$ satisfying (3.3) we have

$$
\frac{2 \int_{0}^{x} e^{-u} u^{3} d u}{\left(\int_{0}^{x} e^{-u} u d u\right)^{2}} \geq \frac{y^{2}}{\left(\int_{0}^{y} e^{-u} u d u\right)}
$$

The function $g, g(y):=y^{2} /\left(\int_{0}^{y} e^{-u} u d u\right)$ is increasing since

$$
2 \int_{0}^{y} e^{y-u} u d u \geq 2 \int_{0}^{y} u d u=y^{2}
$$

i.e.

$$
2 y \int_{0}^{y} e^{-u} u d u \geq e^{-y} y^{3} .
$$

Define $\varphi: \mathbb{R}_{+} \rightarrow \mathbb{R}_{+}$by the requirement

$$
\int_{0}^{\varphi(x)} e^{-u} u d u=\left(\frac{4}{5}\right)^{2} \int_{0}^{x} e^{-u} u d u .
$$

Hence by assumption $y \leq \varphi(x) \leq x$, and (3.4) will follow from the stronger claim

$$
\frac{2 \int_{0}^{x} e^{-u} u^{3} d u}{\left(\int_{0}^{x} e^{-u} u d u\right)^{2}} \geq \frac{\varphi(x)^{2}}{\left(\int_{0}^{\varphi(x)} e^{-u} u d u\right)}=\left(\frac{5}{4}\right)^{2} \frac{\varphi(x)^{2}}{\left(\int_{0}^{x} e^{-u} u d u\right)},
$$

i.e.

$$
\varphi(x)^{2} \leq \frac{32}{25}\left(\int_{0}^{x} e^{-u} u^{3} d u / \int_{0}^{x} e^{-u} u d u\right)=: \psi(x) .
$$

Let $x \in[0,4]$. Since $\varphi(0)=0, \lim _{x \searrow 0} \psi(x)=0$, to prove (3.6) in this range of $x$ it suffices to show that $\left(\varphi(x)^{2}\right)^{\prime} \leq \psi^{\prime}(x)$. We have by (3.5)

$$
\left(\varphi(x)^{2}\right)^{\prime}=2 \varphi(x) \varphi^{\prime}(x)=\frac{32}{25} e^{\varphi(x)} x e^{-x}
$$

and

$$
\psi^{\prime}(x)=\frac{32 / 25}{\left(\int_{0}^{x} e^{-u} u d u\right)^{2}}\left[\left(\int_{0}^{x} e^{-u} u d u\right) x^{3} e^{-x}-x e^{-x}\left(\int_{0}^{x} e^{-u} u^{3} d u\right)\right] .
$$

Hence $\left(\varphi(x)^{2}\right)^{\prime} \leq \psi^{\prime}(x)$ holds provided that

$$
e^{\varphi(x)}\left(\int_{0}^{x} e^{-u} u d u\right)^{2} \leq \int_{0}^{x}\left(x^{2} u-u^{3}\right) e^{-u} d u
$$


or equivalently

$$
\begin{aligned}
e^{\varphi(x)} & \leq f(x) / g(x), f(x):=x^{2}\left(e^{x}+2\right)-6\left(e^{x}-(1+x)\right), \\
g(x) & =\left(e^{x}-(1+x)\right)\left(1-(1+x) e^{-x}\right) .
\end{aligned}
$$

We claim that $\varphi(x) \leq \frac{4}{5} x-\frac{4}{75} x^{2}=: \gamma(x)$ holds. This will follow from

$$
\int_{0}^{\gamma(x)} e^{-u} u d u \geq\left(\frac{4}{5}\right)^{2} \int_{0}^{x} e^{-u} u d u
$$

by definition of $\varphi$. (3.8) will follow from the stronger inequality for the derivatives

$$
\gamma^{\prime}(x) e^{-\gamma(x)} \gamma(x) \geq\left(\frac{4}{5}\right)^{2} e^{-x} x
$$

i.e.

or

$$
\left(1-\frac{2}{15} x\right)\left(1-\frac{1}{15} x\right) e^{x / 5+4 x^{2} / 75} \geq 1
$$

$$
\frac{x}{5}+\frac{4}{75} x^{2}+\ln \left(1-\frac{2}{15} x\right)+\ln \left(1-\frac{1}{15} x\right) \geq 0 .
$$

For $x \leq 4, \frac{2}{15} x \leq \frac{3}{5}$ and for $y \leq \frac{3}{5}, \ln (1-y) \geq-y-y^{2}$, which implies

$$
\ln \left(1-\frac{2}{15} x\right)+\ln \left(1-\frac{1}{15} x\right) \geq-\frac{x}{5}-\frac{x^{2}}{45} .
$$

Hence (3.9) and (3.8) hold for $x \leq 4$. We conclude that

$$
e^{\varphi(x)} \leq e^{4 / 5 x-4 x^{2} / 75} \leq 1+\frac{4}{5} x+\frac{4}{15} x^{2}+\frac{16}{375} x^{3}+\frac{8}{5625} x^{4},
$$

the last inequality coming from the series development of the middle term. As for $f$ and $g$ in (3.7), one calculates the Taylor expansions

$$
\begin{aligned}
& f(x)=\frac{x^{4}}{4} \sum_{n=0}^{\infty} \frac{4(n+1)(n+6)}{(n+4) !} x^{n} \\
& g(x)=\frac{x^{4}}{4} \sum_{n=0}^{\infty}(-1)^{n} \cdot 4 \cdot \frac{(n+4)^{2}-3(n+4)+1+(-1)^{n}}{(n+4) !} x^{n} .
\end{aligned}
$$

The Taylor polynomial for $f(x) / g(x)$ of order 3 yields a lower bound

$$
1+\frac{4}{5} x+\frac{13}{45} x^{2}+\frac{11}{189} x^{3} \leq f(x) / g(x) .
$$

Inequality (3.7) now follows from (3.10), (3.11) and

$$
\frac{4}{15} x^{2}+\frac{16}{375} x^{3}+\frac{8}{5625} x^{4} \leq \frac{13}{45} x^{2}+\frac{11}{189} x^{3},
$$

which is true since $1+\frac{367}{525} x-\frac{8}{125} x^{2} \geq 0$ for $0 \leq x \leq 4$ holds.

For $x \geq 4$, inequality (3.9) is verified more easily: $\varphi$ and $\psi$ are increasing functions with

$$
\lim _{x \rightarrow \infty} \psi(x)=\frac{192}{25}=7.68, \lim _{x \rightarrow \infty} \varphi(x)^{2} \simeq 4,74 .
$$

For $x \geq 4$

$$
\psi(x) \geq \psi(4) \simeq 4.79 \geq \varphi(\infty)^{2} \geq \varphi(x)^{2} .
$$




\section{REFERENCES}

[B] K. Ball; Cube slicing in $\mathbb{R}^{n}$, Proc. AMS 97 (1986), 465-473. MR840631 (87g:60018)

[BK] F. Barthe, A. Koldobsky; Extremal slabs in the cube and the Laplace transform, Advances in Math. 174 (2003), 89-114. MR.1959893 (2004f:52004)

[Ha] H. Hadwiger; Gitterperiodische Punktmengen und Isoperimetrie, Monatsh. Math. 76 (1972), 410-418. MR0324550 (48:2902)

[He] D. Hensley; Slicing the cube in $\mathbb{R}^{n}$ and probability, Proc. AMS 73 (1979), 95-100. MR.512066 (80b:60025)

[OP] K. Oleskiewicz, A. Pełczyński; Polydisc slicing in $\mathbb{C}^{n}$, Studia Math. 142 (2000), 281-294. MR 1792611(2001f:52014)

[P] G. Polya; Berechnung eines bestimmten Integrals, Math. Ann. 74 (1913), 204-212. MR 1511759

[V] J. D. Vaaler; A geometric inequality with applications to linear forms, Pacific J. Math. 83 (1979), 543-553. MR557952 (81d:52007)

[W] G. N. Watson; A treatise on the theory of Bessel functions, Cambridge University Press, 1952. MR1349110 (96i:33010)

Mathematisches Seminar, Universität zu Kiel, 24098 Kiel, Germany

E-mail address: hkoenig@math.uni-kiel.de

Department of Mathematics, University of Missouri, Columbia, Missouri 65211

E-mail address: koldobsk@math.missouri.edu 